\title{
RKKY interaction in three-dimensional electron gases with linear spin-orbit coupling
}

\author{
Shi-Xiong Wang, ${ }^{1}$ Hao-Ran Chang,,${ }^{1,2, *}$ and Jianhui Zhou ${ }^{3, \dagger}$ \\ ${ }^{1}$ Department of Physics, Institute of Solid State Physics, and Center for Computational Sciences, \\ Sichuan Normal University, Chengdu, Sichuan 610066, China \\ ${ }^{2}$ Department of Physics, McGill University, Montreal, Quebec H3A 2T8, Canada \\ ${ }^{3}$ Department of Physics, The University of Hong Kong, Pokfulam Road, Hong Kong, China \\ (Received 5 July 2017; revised manuscript received 15 August 2017; published 13 September 2017)
}

\begin{abstract}
We theoretically study the impacts of linear spin-orbit coupling (SOC) on the Ruderman-Kittel-Kasuya-Yosida interaction between magnetic impurities in two kinds of three-dimensional noncentrosymmetric systems. It has been found that linear SOCs lead to the Dzyaloshinskii-Moriya interaction and the Ising interaction, in addition to the conventional Heisenberg interaction. These interactions possess distinct range functions from three-dimensional electron gases and Dirac/Weyl semimetals. In the weak SOC limit, the Heisenberg interaction dominates over the other two interactions in a moderately large region of parameters. Sufficiently strong Rashba SOC makes the Dzyaloshinskii-Moriya interaction or the Ising interaction dominate over the Heisenberg interaction in some regions. The change in topology of the Fermi surface leads to some quantitative changes in periods of oscillations of range functions. The anisotropy of Ruderman-Kittel-Kasuya-Yosida interaction in bismuth tellurohalides family $\mathrm{BiTe} X(X=\mathrm{Br}, \mathrm{Cl}$, and I) originates from both the specific form of Rashba SOC and the anisotropic effective mass. Our work provides some insights into understanding observed spin textures and the application of these materials in spintronics.
\end{abstract}

DOI: 10.1103/PhysRevB.96.115204

\section{INTRODUCTION}

Inversion symmetry may be broken at the surface or interface, and the resulting electric fields entangle momentum of an electron to its spin, leading to the Rashba spin-orbit coupling (SOC) [1]. The Rashba SOC has mostly been studied in two-dimensional electron or hole gases in semiconductor heterostructures [2], the surface states of conventional metals [3], and even the two-dimensional electron gases (2DEG) at the interface or surfaces of complex oxides [4-9]. The Rashba SOC, $H_{R}=\alpha_{R}(\boldsymbol{\sigma} \times \boldsymbol{k}) \cdot \hat{z}$, plays a central role in a vast number of phenomena in condensed matter physics, such as spin Hall effect, spin galvanic effect, spin-orbit torques, chiral magnetic textures, topological phases of matter, and Majorana fermions [10-13]. In particular, the interplay between SOC and magnetism is of increasing importance. Rashba SOC could induce a chiral Dzyaloshinskii-Moriya (DM) interaction $[14,15]$, which leads to spin helix, chiral domain walls, and magnetic skyrmions. In addition, Ruderman-Kittel-KasuyaYosida (RKKY) interaction, an indirect exchange interaction between two magnetic impurities mediated by conduction electrons, may provide an alternative mechanism for the DM interaction. The strength of RKKY interaction, characterized by the range function, usually oscillates as a function of distance between magnetic impurities with the period determined by the Fermi level, the effective mass of conduction electrons, and other material-dependent parameters [16-18].

Recently, the Rashba SOC in three-dimensional (3D) systems lacking inversion symmetry has been receiving significant interest $[19,20]$. In the bismuth tellurohalides family $\operatorname{BiTe} X(X=\mathrm{Br}, \mathrm{Cl}$, and $\mathrm{I})$, the structure inversion asymmetry results from the asymmetric stacking of $\mathrm{Bi}, \mathrm{Te}$, and $X$

\footnotetext{
*hrchang@mail.ustc.edu.cn

†jianhuizhou1@gmail.com
}

layers, in which an exceptionally large Rashba parameter had been revealed by angle-resolved photoemission spectroscopy measurements [21,22]. For example, the strength of Rashba SOC reaches the value of 3.9-4.3 eVA for BiTeI as large as that of the surface state of topological insulators [22]. Symmetry analysis suggests that the conduction electrons in $B_{20}$ compounds (a class of cubic helimagnets) [23] and noncentrosymmetric metal $\mathrm{Li}_{2}\left(\mathrm{Pd}_{1-x}, \mathrm{Pt}_{x}\right)_{3} \mathrm{~B}$ [24] possess a strong SOC of the form $\boldsymbol{k} \cdot \boldsymbol{\sigma}$. Many nontrivial spin textures have already been observed in $\operatorname{BiTe} X[21,25,26]$ as well as $B_{20}$ compounds [27-30]. To thoroughly understand the observed various interesting spin textures and to engineer exotic states of quantum matter, we would like to systematically study the effects of linear SOCs on the RKKY interaction.

In this paper, we find that 3D linear SOCs give rise to the DM interaction, the Ising interaction, and the Heisenberg interaction, which possess entirely different range functions from those of 3D electron gases (3DEG) and Dirac/Weyl semimetals. In the weak SOC limit, the Heisenberg interaction dominates over the other two interactions for a relatively large region of parameters. As the Rashba SOC increases, sufficiently strong Rashba SOC makes the DM interaction or the Ising interaction more favorable than the Heisenberg interaction. As the topology of the Fermi surface changes, the periods of oscillations of range functions vary accordingly. In the family $\mathrm{BiTe} X$, both the Rashba SOC and the anisotropic effective mass contribute to the anisotropy of the RKKY interaction.

The rest of this paper is organized as follows. In Sec. II, we outline the formalism for the RKKY interaction. In Sec. III, we derive the exact analytical expressions of RKKY interactions for noncentrosymetric metals and the approximate expressions in weak SOC limit and in long-range limit. In Sec. IV, we reveal the general features of RKKY interactions for 3D Rashba semiconductors numerically and find the analytical expressions in weak Rashba SOC limit. In Sec. V, the main 
results of this paper are summarized. Finally, in the appendices, we give the detailed calculations of range functions of the RKKY interaction.

\section{RKKY INTERACTION AND SPIN-ORBIT COUPLING}

The RKKY interaction is an indirect exchange interaction between two localized spins via the spin polarization of conduction electrons [16-18]. In recent years, it has been shown that the RKKY interaction plays an important role in giant magnetoresistance in multilayer structures [31], ferromagnetism in diluted magnetic semiconductors [32], topological phases, and Majorana fermions [11]. We restrict ourselves to a pair of magnetic impurities, $\boldsymbol{S}_{1}$ and $\boldsymbol{S}_{2}$ located at $\boldsymbol{R}_{1}$ and $\boldsymbol{R}_{2}$, respectively. We assume the interaction between magnetic impurities and conduction electrons is described by the standard $s-d$ interaction [33]:

$$
H_{s-d}=J \sum_{l=1,2} \boldsymbol{S}_{l} \cdot \boldsymbol{\sigma} \delta\left(\boldsymbol{r}-\boldsymbol{R}_{l}\right),
$$

where $\sigma=\left(\sigma_{x}, \sigma_{y}, \sigma_{z}\right)$ denotes for the vector of Pauli spin matrices and $J$ refers to the strength of the $s-d$ interaction. At zero temperature the indirect exchange interaction between these two localized spins mediated by itinerant electrons is given by [34]

$$
\begin{aligned}
H_{\mathrm{RKKY}}= & -\frac{J^{2}}{\pi} \operatorname{Im} \int_{-\infty}^{\varepsilon_{F}} \mathrm{~d} \varepsilon \operatorname{Tr}\left[\left(\boldsymbol{S}_{1} \cdot \boldsymbol{\sigma}\right) G\left(\boldsymbol{R} ; \varepsilon+i 0^{+}\right)\right. \\
& \left.\times\left(\boldsymbol{S}_{2} \cdot \boldsymbol{\sigma}\right) G\left(-\boldsymbol{R} ; \varepsilon+i 0^{+}\right)\right],
\end{aligned}
$$

where $\boldsymbol{R}=\boldsymbol{R}_{2}-\boldsymbol{R}_{1}, \varepsilon_{F}$ is the Fermi energy, and Tr represents the trace over the spin degree of freedom of itinerant electrons. The Green's functions in the energy-coordinate representation can be obtained from the Fourier transform:

$$
G\left( \pm \boldsymbol{R} ; \varepsilon+i 0^{+}\right)=\int \frac{\mathrm{d}^{3} \boldsymbol{k}}{(2 \pi)^{3}} G\left(\boldsymbol{k} ; \varepsilon+i 0^{+}\right) \exp ( \pm i \boldsymbol{k} \cdot \boldsymbol{R}),
$$

where $G^{-1}\left(\boldsymbol{k} ; \varepsilon+i 0^{+}\right)=\varepsilon+i 0^{+}-H_{0}(\boldsymbol{k})$ is the inverse of momentum-space Green's function, $H_{0}(\boldsymbol{k})$ is the Hamiltonian for the noninteracting conduction electrons, and $0^{+}$is a positive infinitesimal.

The specific expressions of the RKKY interactions strongly depend on the spatial dimensionality of systems $[35,36]$, energy dispersion of the conduction carriers, and the property of Rashba SOC. For instance, the RKKY interaction for 3D Dirac electrons with linear dispersion in the context of Dirac/Weyl semimetals $[34,37,38]$ greatly differs from that of the conventional 3DEG [16-18]. The influence of the Rashba SOC had been extensively examined in both one-dimensional electron gases (1DEG) [39-42] and 2DEG [39,43-47]. In the present paper, we shall focus on the impacts of linear SOC on the RKKY interactions in both 3D noncentrosymmetric metals and Rashba semiconductors $\operatorname{BiTe} X$. In the former, each component of spin is parallel to momentum and forms hedgehog spin texture, as shown in Figs. 1(a) and 1(b). In the latter, for a given $k_{z}$, the in-plane components of spin and momenta are perpendicular to each other as the manner of 2DEG with linear Rashba SOC [see Figs. 1(c) and 1(d)]. Note that the topology of the Fermi surface changes at $\varepsilon_{F}=0$.

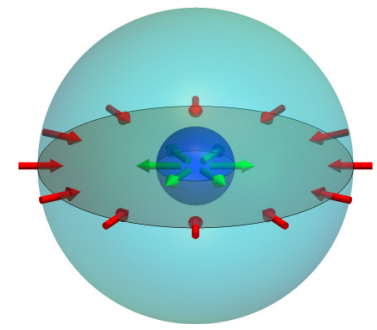

(a)

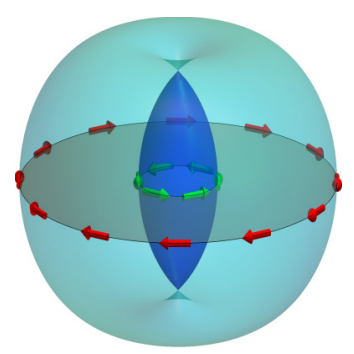

(c)

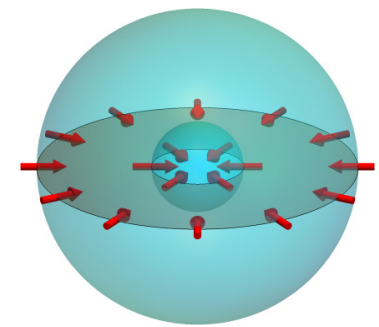

(b)

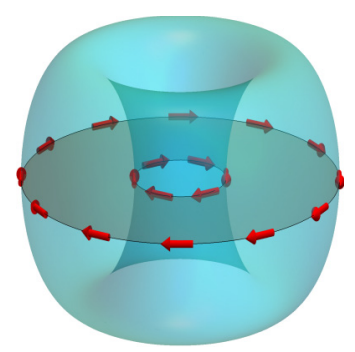

(d)
FIG. 1. Distinct spin textures at Fermi surfaces of 3D noncentrosymmetric metals (a) and (b) and 3D Rashba semiconductors (c) and (d). For (a) and (c), the Fermi energy is positive $\varepsilon_{F}>0$, while for (b) and (d), $\varepsilon_{F}<0$. The green and red arrows show the spin directions at the Fermi energy for a given $k_{z}$ as a function of the two momenta, $k_{x}$ and $k_{y}$. The topology of Fermi surface changes at $\varepsilon_{F}=0$ from two Fermi surfaces with different spin directions in (a) and (c) to a closed Fermi surface in (b) and (d) or vice versa.

\section{THREE-DIMENSIONAL NONCENTROSYMMETRIC METALS}

Three-dimensional noncentrosymmetric metals, such as $B_{20}$ compounds [23,48] and the $\mathrm{Li}_{2}\left(\mathrm{Pd}_{1-x}, \mathrm{Pt}_{x}\right)_{3} \mathrm{~B}$ family [49], can be effectively described by the following Hamiltonian:

$$
H_{0}^{\mathrm{NCS}}=\frac{k^{2}}{2 m}+\alpha \boldsymbol{k} \cdot \boldsymbol{\sigma},
$$

and the energy dispersion reads

$$
\varepsilon_{\lambda k}=\frac{k^{2}}{2 m}+\lambda \alpha k
$$

where $m$ is the effective mass, $\boldsymbol{k}=\left(k_{x}, k_{y}, k_{z}\right)$, and $k=$ $\sqrt{k_{x}^{2}+k_{y}^{2}+k_{z}^{2}} \cdot \lambda= \pm 1$ denotes for the two energy bands with opposite chirality, $\alpha>0$ represents the strength of SOC. The superscript NCS stands for the noncentrosymmetric metals. For a positive Fermi energy $\varepsilon_{F}>0$, there are two distinct Fermi wave vectors $k_{F \pm}=\mp m \alpha+\sqrt{m^{2} \alpha^{2}+2 m \varepsilon_{F}}$, characterizing the two Fermi surfaces in Fig. 1(a). On the other hand, for $-m \alpha^{2} / 2<\varepsilon_{F}<0$, only one closed Fermi surface exists in Fig. 1(b), but is characterized by two Fermi wave vectors $k_{F \pm}=m \alpha \pm \sqrt{m^{2} \alpha^{2}+2 m \varepsilon_{F}}>0$. As will be shown below, the change in topology of the Fermi surface leads to some quantitative modifications to the RKKY interactions [see Figs. 2(c) and 2(d)]. 

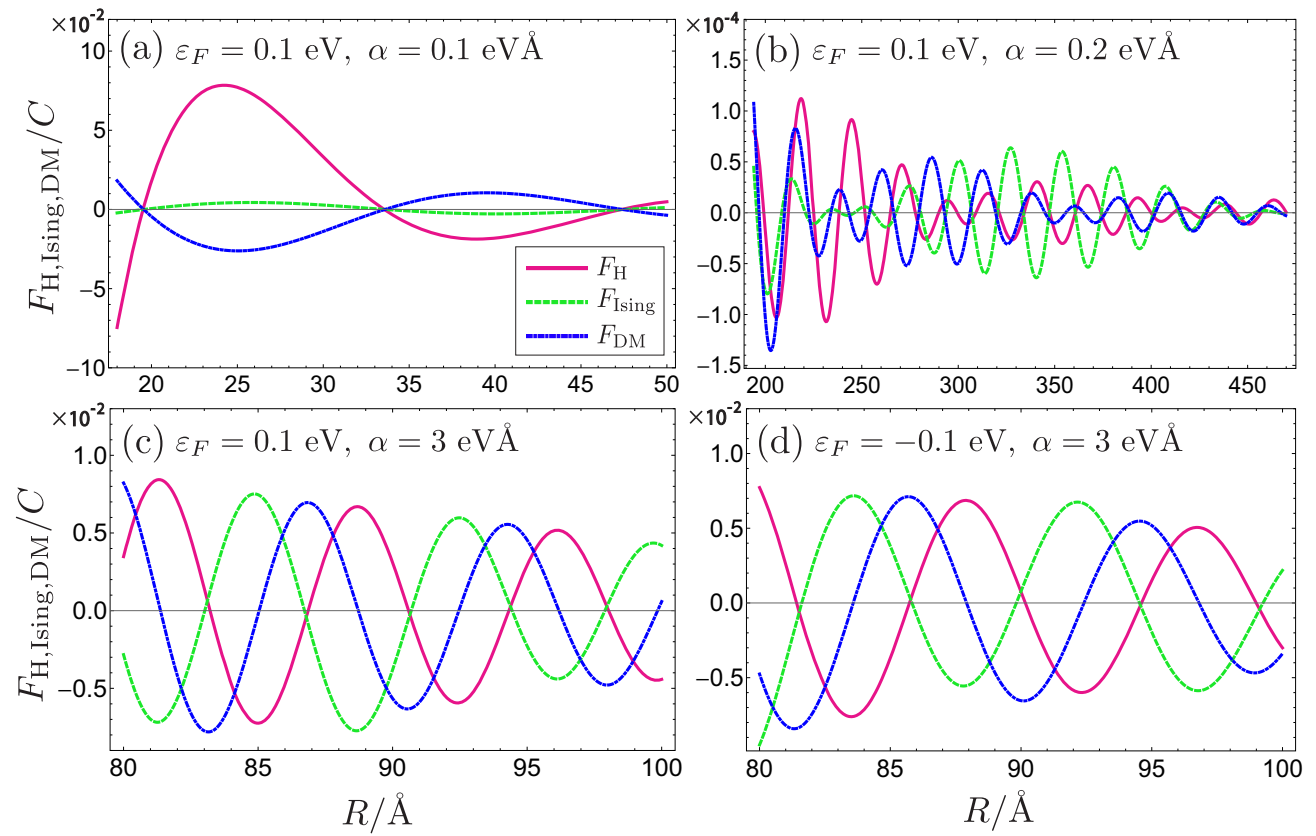

FIG. 2. The exact range functions of RKKY interactions in 3D noncentrosymmetric metals for different values of SOCs. The change in topology of the Fermi surface leads to quantitative changes in the periods of oscillations (c) and (d). The effective mass is equal to half of the electron rest mass.

After some straightforward calculations, one gets the corresponding Berry curvatures of Bloch electrons as

$$
\boldsymbol{\Omega}_{ \pm}(\boldsymbol{k})=\mp \frac{\boldsymbol{k}}{2 k^{3}},
$$

which can be viewed as effective magnetic monopoles in the momentum space, similar to those in 3D Weyl semimetals [50]. One thus expects that the electrons in noncentrosymmetric metals inherit the properties of electrons from both the conventional 3DEG and the Dirac/Weyl semimetals [51].

According to Eq. (4), one directly evaluates the Green's function in the momentum space as

$$
G\left(\boldsymbol{k} ; \varepsilon+\mathrm{i} 0^{+}\right)=\frac{1}{2} \sum_{\lambda= \pm} \frac{\sigma_{0}+\lambda \hat{\boldsymbol{k}} \cdot \boldsymbol{\sigma}}{\varepsilon-\varepsilon_{\lambda \boldsymbol{k}}+i 0^{+}},
$$

where $\hat{\boldsymbol{k}}=\boldsymbol{k} / k$ denotes for the unit vector parallel to $\boldsymbol{k}$ and $\sigma_{0}$ refers to the identity matrix. Due to the rotational symmetry of itinerant electrons described by Eq. (4), without loss of generality, we align the two magnetic impurities with the $j$ axis, i.e., $\boldsymbol{R}=R \boldsymbol{e}_{j}$, with $R$ being the distance between two magnetic impurities. After carrying out Fourier transform, we decompose the real-space Green's function into two parts as

$$
G\left( \pm \boldsymbol{R} ; \varepsilon+i 0^{+}\right)=G_{0} \sigma_{0} \pm G_{R} \sigma_{j},
$$

where the functions are given as

$$
\begin{aligned}
G_{0} & =\frac{-m \exp (i \xi)}{2 \pi R\left(\xi+\mathrm{i} 0^{+}\right)}(\xi \cos \zeta+i \zeta \sin \zeta), \\
G_{R} & =\frac{m \exp (i \xi)}{2 \pi R\left(\xi+i 0^{+}\right)}[(i \xi-1) \sin \zeta+\zeta \cos \zeta],
\end{aligned}
$$

with two dimensionless parameters $\zeta=m \alpha R$ and $\xi=$ $\sqrt{m^{2} \alpha^{2}+2 m \varepsilon} R$. The second term $G_{R} \sigma_{j}$ in Eq. (8) comes from the Rashba SOC and greatly modifies the RKKY interactions. Consequently, the RKKY interaction contains three terms: Heisenberg interaction, Ising interaction, and DM interaction:

$$
H_{\mathrm{RKKY}}^{\mathrm{NCS}}=F_{\mathrm{H}} \boldsymbol{S}_{1} \cdot \boldsymbol{S}_{2}+F_{\text {Ising }} S_{1}^{j} S_{2}^{j}+F_{\mathrm{DM}}\left(S_{1} \times S_{2}\right)_{j},
$$

where these three range functions are given by

$$
\begin{aligned}
F_{\mathrm{H}} & =-\frac{2 J^{2}}{\pi} \operatorname{Im} \int_{-\infty}^{\varepsilon_{F}}\left(G_{0}^{2}+G_{R}^{2}\right) \mathrm{d} \varepsilon, \\
F_{\text {Ising }} & =\frac{4 J^{2}}{\pi} \operatorname{Im} \int_{-\infty}^{\varepsilon_{F}} G_{R}^{2} \mathrm{~d} \varepsilon, \\
F_{\mathrm{DM}} & =\frac{4 J^{2}}{\pi} \operatorname{Im} \int_{-\infty}^{\varepsilon_{F}} i G_{0} G_{R} \mathrm{~d} \varepsilon .
\end{aligned}
$$

The Rashba SOC has two obvious impacts on the RKKY interaction. First, it modifies the Heisenberg interaction in Eq. (11). Second, both the Ising interaction and the DM interaction entirely originate from the Rashba SOC. It should be emphasized that the DM vector along $\boldsymbol{R}=\boldsymbol{R} \boldsymbol{e}_{j}$ is compatible with the symmetry of $B_{20}$ compounds [23]. Therefore, the RKKY interaction here may provide us an alternative physical origin for the DM interaction, which is essential for the chiral spin textures [27-30]. Physically, the cross-product nature of the DM interaction implies that an inversion operation about the center of the joint line would exchange two neighboring spins such that the DM interaction flips sign. The DM interaction would disappear if the two localized spins are parallel or antiparallel. However, the Heisenberg interaction and Ising interaction tend to align neighboring spins and are unchanged under this operation, respecting the inversion symmetry.

After performing these complex integrals, one has the exact analytical range functions in Eq. (11) (the detailed calculations 
can be found in Appendix A):

$$
\begin{aligned}
F_{\mathrm{H}}\left(\xi_{F}, \zeta\right)= & \frac{C}{\left(k_{F} R\right)^{4}}\left\{2 \xi_{F} \cos (2 \zeta) \cos \left(2 \xi_{F}\right)\right. \\
& -[4 \zeta \sin (2 \zeta)+3 \cos (2 \zeta)-2] \sin \left(2 \xi_{F}\right) \\
& \left.-2\left[2 \zeta^{2} \cos (2 \zeta)+g(\zeta)\right] \operatorname{si}\left(2 \xi_{\mathrm{F}}\right)\right\}, \\
F_{\text {Ising }}\left(\xi_{F}, \zeta\right)= & \frac{C}{\left(k_{F} R\right)^{4}}\left\{2 \xi_{F}[1-\cos (2 \zeta)] \cos \left(2 \xi_{F}\right)\right. \\
& +[4 \zeta \sin (2 \zeta)+5 \cos (2 \zeta)-5] \sin \left(2 \xi_{F}\right) \\
& \left.+4\left[\zeta^{2}(\cos (2 \zeta)+1)+g(\zeta)\right] \operatorname{si}\left(2 \xi_{F}\right)\right\}, \\
F_{\mathrm{DM}}\left(\xi_{F}, \zeta\right)= & \frac{-C}{\left(k_{F} R\right)^{4}}\left\{2 \xi_{F} \sin (2 \zeta) \cos \left(2 \xi_{F}\right)\right. \\
& +[4 \zeta \cos (2 \zeta)-3 \sin (2 \zeta)] \sin \left(2 \xi_{F}\right) \\
& \left.-4\left[\zeta^{2} \sin (2 \zeta)+\zeta(\cos (2 \zeta)-1)\right] \operatorname{si}\left(2 \xi_{F}\right)\right\},
\end{aligned}
$$

where $\quad g(x)=-2 x \sin (2 x)-\cos (2 x)+1, \quad \xi_{F}=$ $\sqrt{m^{2} \alpha^{2}+2 m \varepsilon_{F}} R, \quad k_{F}=\sqrt{2 m\left|\varepsilon_{F}\right|}$ is the Fermi wave vector in the absence of SOC, $\operatorname{si}(z)=-\int_{z}^{\infty} \mathrm{d} t \sin t / t$ is the sine integral function, and $C=J^{2} m k_{F}^{4} /(2 \pi)^{3}$. The three range functions in Eq. (12) exhibit several features. First, as shown in Fig. 2(a), for a weak SOC, the Heisenberg interaction dominates over the other two interactions within a moderately long range. Second, the magnitudes of Fermi wave vectors of these two Fermi surfaces are unequal $k_{F-}-k_{F+}=2 m \alpha$ for $\varepsilon_{F}>0$ or $k_{F+}-k_{F-}=2 \sqrt{m^{2} \alpha^{2}+2 m \varepsilon_{F}}$ for $\varepsilon_{F}<0$ such that the range functions in Eq. (12) oscillate with two distinct periods and form beating patterns in Fig. 2(b). Third, while the range functions for $\varepsilon_{F}<0$ share similar features to those for $\varepsilon_{F}>0$, Figs. 2(c) and 2(d) clearly show that the oscillation period quantitatively differs between the two cases. In addition, Fig. 2 shows that these range functions display a damped oscillatory behavior with increasing distance $R$, with each term dominating in different regions of the parameters $\varepsilon_{F}, R$, and $\alpha$. The competition among these interactions leads to rich spin textures, which can be detected by a variety of experimental tools.

To gain more insight into the impacts of SOC on the RKKY interactions, let us examine the weak SOC limit $\alpha k_{F} / \varepsilon_{F} \ll 1$. Expanding the analytical expressions of range functions in Eq. (12) in power of $\alpha$ and keeping the correction up to $\alpha^{2}$, one finally gets the following approximate range functions:

$$
\begin{aligned}
F_{\mathrm{H}} & \simeq 4 F_{0}(R)-4 C \zeta^{2} h\left(k_{F} R\right), \\
F_{\text {Ising }} & \simeq 8 F_{0}(R) \zeta^{2}, \\
F_{\mathrm{DM}} & \simeq-8 F_{0}(R) \zeta,
\end{aligned}
$$

where $h(x)=[x \cos (2 x)+\sin (2 x)] / x^{4}, F_{0}(R)$ is the range function for the conventional 3DEG [16-18]:

$$
F_{0}(R)=\frac{C\left[2 k_{F} R \cos \left(2 k_{F} R\right)-\sin \left(2 k_{F} R\right)\right]}{4\left(k_{F} R\right)^{4}} .
$$

According to Eq. (13), the Ising interaction and DM interaction are quadratic and linear in $\zeta$, respectively. Thus, in the weak SOC limit $\alpha k_{F} / \varepsilon_{F} \ll 1$, the Heisenberg interaction becomes dominant as that of the 3DEG. It qualitatively coincides with the behavior of the exact range functions depicted in Fig. 2(a).

When the magnetic impurities are dilute, the RKKY interaction is mainly controlled by its long-range behavior. Let us turn to the long-range case of $\xi_{F} \gg 1$ and $\xi_{F} \gg \zeta$, which are equivalent to $R \gg 1 / \sqrt{m^{2} \alpha^{2}+2 m \varepsilon_{F}}$ and $\sqrt{m^{2} \alpha^{2}+2 m \varepsilon_{F}} \gg$ $m \alpha$, respectively. Under these conditions, we have $\operatorname{si}\left(2 \xi_{F}\right) \simeq$ $-\cos \left(2 \xi_{F}\right) / 2 \xi_{F}$ and hence $\xi_{F}\left|\cos \left(2 \xi_{F}\right)\right| \gg \zeta\left|\cos \left(2 \xi_{F}\right)\right| \gg$ $\zeta^{2}\left|\operatorname{si}\left(2 \xi_{F}\right)\right|$. As a result, the long-range RKKY interaction can be cast into a simple twisted form:

$$
H_{\mathrm{RKKY}}^{\mathrm{NCS}} \simeq \frac{2 C \xi_{F} \cos \left(2 \xi_{F}\right)}{\left(k_{F} R\right)^{4}} \boldsymbol{S}_{1} \cdot \tilde{\boldsymbol{S}}_{2},
$$

where $\tilde{\boldsymbol{S}}_{2}$ stands for the twisted spin operator of the second localized magnetic impurity and can be obtained from a rotation in spin space around the $j$ axis (the direction of $\boldsymbol{R}$ ) by an angle $2 \zeta$ [52]. For example, taking $\boldsymbol{R}=R \boldsymbol{e}_{x}$, after such a rotation the $x$ component of $\boldsymbol{S}_{2}$ remains unchanged, but the other two components become $\tilde{S}_{2}^{y}=\cos (2 \zeta) S_{2}^{y}-\sin (2 \zeta) S_{2}^{z}$ and $\tilde{S}_{2}^{z}=\sin (2 \zeta) S_{2}^{y}+\cos (2 \zeta) S_{2}^{z}$. It is clear that the SOC tends to twist the spin to form a collinear coupling of localized spins. This kind of twisted exchange interaction was also discussed in the context of 1DEG and 2DEG with standard Rashba SOC. However, their range functions essentially differ from ours. Specifically, the corresponding range functions in $1 \mathrm{D}$ and $2 \mathrm{D}$ are proportional to $\operatorname{si}\left(2 \xi_{F}\right)$ and $\sin \left(2 \xi_{F}\right) / R^{2}$, respectively [39]. In addition, the range function in Eq. (15) is the same as that of 3DEG at large distance by replacing $k_{F} R$ with $\xi_{F}$, up to a degeneracy factor. Furthermore, it should be noted that the spatial dependence of $R^{-3}$ is the same as both 3DEG [35] and 3D Dirac/Weyl semimetals [34,37,38].

\section{THREE-DIMENSIONAL RASHBA SEMICONDUCTORS}

The electrons in 3D Rashba semiconductors BiTe $X$ can be described by the following effective Hamiltonian [21,53,54]:

$$
H_{0}^{3 \mathrm{DR}}=\frac{k_{x}^{2}+k_{y}^{2}}{2 m}+\frac{k_{z}^{2}}{2 m_{3}}+\alpha_{R}(\boldsymbol{\sigma} \times \boldsymbol{k})_{z},
$$

and the corresponding energy dispersion is given as

$$
\epsilon_{\lambda k}=\frac{k_{x}^{2}+k_{y}^{2}}{2 m}+\frac{k_{z}^{2}}{2 m_{3}}+\lambda \alpha_{R} \sqrt{k_{x}^{2}+k_{y}^{2}},
$$

where the superscript 3DR refers to the 3D Rashba semiconductors, $\lambda= \pm$ stands for the chirality of energy bands, $\alpha_{R}$ is the strength of Rashba SOC, and $m$ and $m_{3}$ denote for the effective masses in the $x-y$ plane and along the $z$ direction, respectively. A series of experiments show that the ratio of $m_{3} / m$ ranges from 5 to 10. As shown in Fig. 1(c), for $\varepsilon_{F}>0$, there are two distinct Fermi surfaces (spindle torus), whereas only one closed Fermi surface (ring torus) exists for $\varepsilon_{F}<0$ [25]. This topological transition of the bulk Fermi surface as a function of the Fermi energy had been detected through Shubnikov-de Haas quantum oscillation [55,56] and thermoelectric effects [57]. The kinds of semiconductors described by Eq. (16) can be intuitively seen as a coherent superposition of layers of $2 \mathrm{DEG}$ with Rashba SOC along the $z$-direction. Unlike noncentrosymmetric metals, the Rashba SOC in Eq. (16) only entangles $k_{x}$ and $k_{y}$ with $\sigma_{y}$ and $\sigma_{x}$, respectively, but leaves $k_{z}$ and $\sigma_{z}$ free. 
Therefore, the magnetism for in-plane magnetic impurities should significantly differ from that for out-plane ones.

In the bismuth tellurohalides family $\mathrm{BiTe} X(X=\mathrm{Br}, \mathrm{Cl}, \mathrm{I})$, the structure inversion asymmetry results from the asymmetric stacking of $\mathrm{Bi}, \mathrm{Te}$, and $X$ layers, in which an exceptionally large Rashba parameter and $\pi$ Berry phase [50] had been revealed by photoemission studies [21] and quantum oscillation experiment [59], respectively. The strengths of Rashba SOC have been obtained experimentally: $3.9-4.3 \mathrm{eVA}$ for BiTeI, 2.0-2.1 eVA for BiTeBr, and 1.7-2.2 eVA for BiTeCl [22].

We shall consider the general case with the relative position vector between magnetic impurities $\boldsymbol{R}=R_{x} \boldsymbol{e}_{x}+R_{y} \boldsymbol{e}_{y}+$ $R_{z} \boldsymbol{e}_{z}$. Following the similar procedure, one gets the RKKY interaction as

$$
\begin{aligned}
H_{\mathrm{RKKY}}^{3 \mathrm{DR}}= & \mathcal{F}_{\mathrm{H}} \boldsymbol{S}_{1} \cdot \boldsymbol{S}_{2}+\mathcal{F}_{\mathrm{I} \operatorname{sing}}\left(S_{1}^{x} \sin \varphi-S_{1}^{y} \cos \varphi\right) \\
& \times\left(S_{2}^{x} \sin \varphi-S_{2}^{y} \cos \varphi\right)+\mathcal{F}_{\mathrm{DM}} \\
& \times\left[\left(\boldsymbol{S}_{1} \times \boldsymbol{S}_{2}\right)_{x} \sin \varphi-\left(\boldsymbol{S}_{1} \times \boldsymbol{S}_{2}\right)_{y} \cos \varphi\right],
\end{aligned}
$$

where these range functions have the same form as those in Eq. (11) but different real-space Green's function $G( \pm \boldsymbol{R}$; $\left.\varepsilon+i 0^{+}\right)=G_{0}\left(R ; \varepsilon+i 0^{+}\right) \sigma_{0} \pm G_{R}\left(R ; \varepsilon+i 0^{+}\right)\left(\sigma_{x} \sin \varphi-\right.$ $\left.\sigma_{y} \cos \varphi\right)$ with $\varphi=\arctan \left(R_{y} / R_{x}\right)$. In contrast to the noncentrosymmetric metals, the DM vector in Eq. (18) lies in the $x-y$ plane and is perpendicular to the line connecting two magnetic impurities.

Direct numerical calculations of the range functions in Eq. (18) show several main features of the RKKY interactions of 3D Rashba semiconductors [58]. First, for a positive Fermi energy $\varepsilon_{F}>0$ and a weak Rashba SOC, the Heisenberg interaction dominates over the other two interactions within a relatively large range in Fig. 3(a). Second, as the Rashba SOC increases, the Ising interaction and DM interaction would be enhanced gradually. Eventually, these three terms compete with each other. In some regions, the Ising interaction or the DM interaction dominates over the Heisenberg interaction, as shown in Figs. 3(b) and 3(c). Third, Figs. 4(a)-4(c) show that these three interactions also compete with each other as the Fermi energy varies. For a small $\varepsilon_{F}$ and a strong Rashba SOC, the Heisenberg interaction is greatly suppressed within a large range of distance. In addition, Fig. 4(d) depicts change in the periods of oscillation of the Ising interaction as the Fermi energy varies. In other words, the magnetism of magnetic impurities can be effectively manipulated through tuning the carrier concentration.

Due to the great complexity of general expressions of the range functions, in the present paper, we mainly focus on the analytical expressions in the weak Rashba SOC case $\alpha_{R} k_{F} / \varepsilon_{F} \ll 1$ and perturbatively treat the Rashba SOC. Keeping the contribution of Rashba SOC up to $\alpha_{R}^{2}$, one arrives at the approximate range functions (the detailed calculations are given in Appendix B):

$$
\begin{aligned}
\mathcal{F}_{\mathrm{H}} \simeq & 4 \gamma^{2} F_{0}(\tilde{R})-8 \gamma^{2} m^{2} \alpha_{R}^{2} \\
& \times\left[\left(R_{x}^{2}+R_{y}^{2}\right) F_{0}(\tilde{R})+\frac{C \sin \left(2 k_{\mathrm{F}} \tilde{R}\right)}{2 k_{\mathrm{F}}^{4} \tilde{R}^{2}}\right], \\
\mathcal{F}_{\text {Ising }} \simeq & 8 \gamma^{2} m^{2} \alpha_{R}^{2}\left(R_{x}^{2}+R_{y}^{2}\right) F_{0}(\tilde{R}), \\
\mathcal{F}_{\mathrm{DM}} \simeq & -8 \gamma^{2} m \alpha_{R} \sqrt{R_{x}^{2}+R_{y}^{2}} F_{0}(\tilde{R}),
\end{aligned}
$$
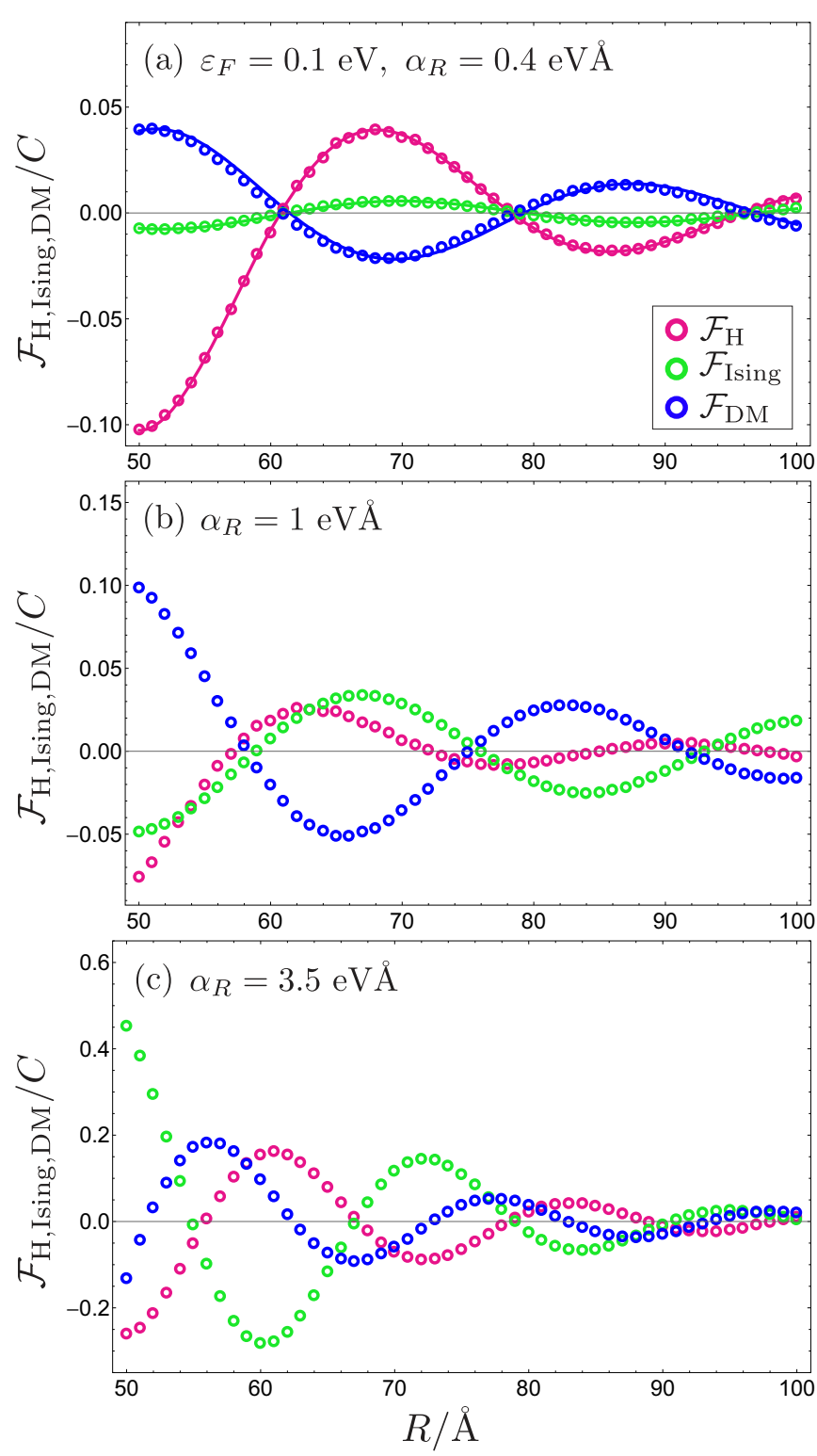

FIG. 3. RKKY interactions of 3D Rashba semiconductors BiTe $X$ for different strengths of Rashba SOCs. (a) shows that the Heisenberg interaction dominates over the Ising and DM interactions within a large range. The solid lines in (a) correspond to the approximate range functions at zero temperature. For a strong Rashba SOC, these three interactions compete with each other in (b) and (c). Other parameters are given in Ref. [58].

where $\tilde{R}=\sqrt{R_{x}^{2}+R_{y}^{2}+\gamma^{2} R_{z}^{2}}$, and $\gamma=\sqrt{m_{3} / m}$ measures the anisotropy of the effective mass. One immediately recognizes that the anisotropic effective mass may result in an anisotropic RKKY interaction. In addition, both sets of range functions in Eq. (13) and Eq. (19) share a similar dependence on the strength of SOC. It should be noted that the approximate range functions are consistent with the exact numerical results (hollow circles) with a small Rashba SOC, as shown in Fig. 3(a). It implies that the low finite temperature only leads to small quantitative modifications.

More interestingly, when the two magnetic impurities locate on a line perpendicular to the $x-y$ plane $\left(\boldsymbol{R}=R_{z} \boldsymbol{e}_{z}\right)$, only the 

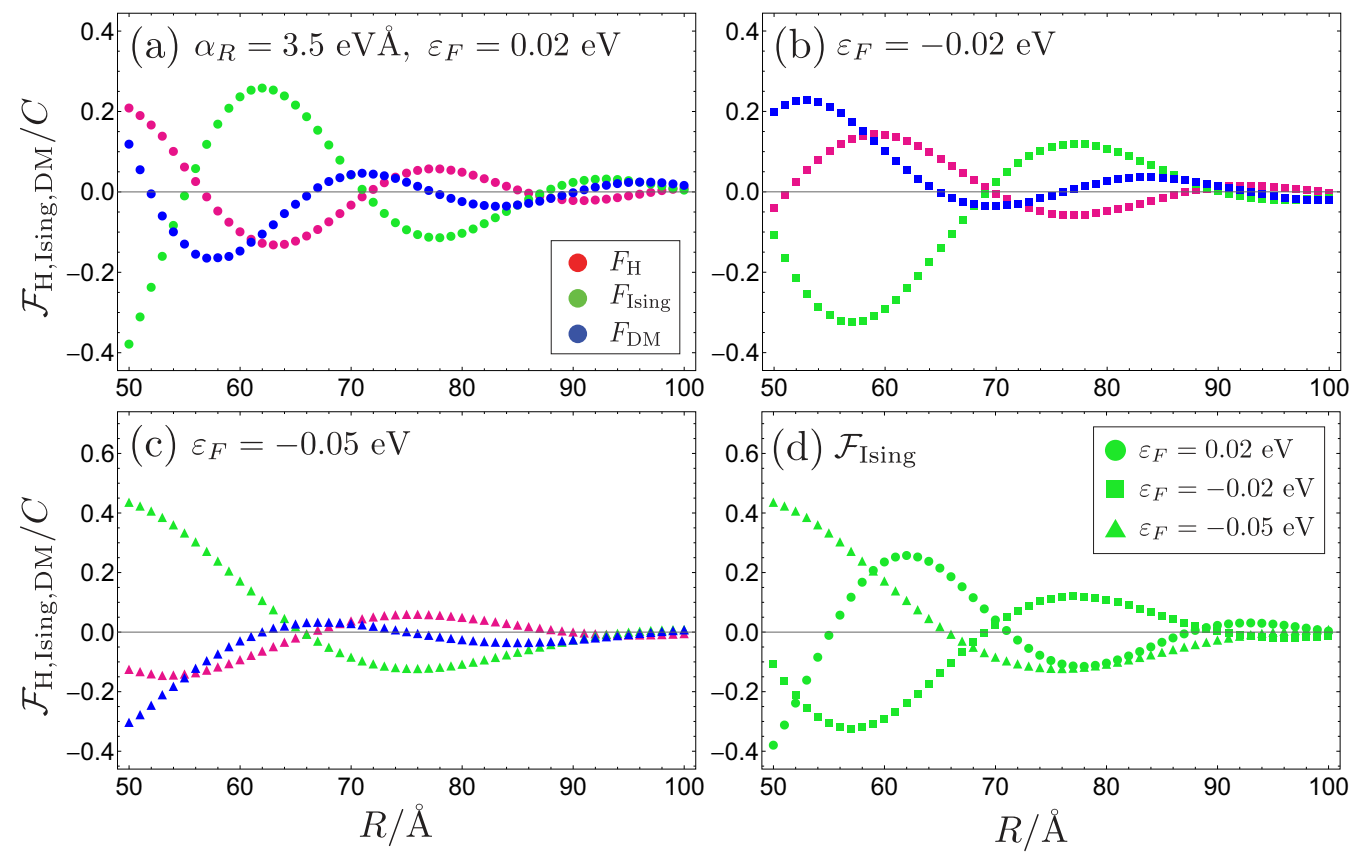

FIG. 4. The evolution of RKKY interactions of 3D Rashba semiconductors BiTe $X$ for different values of Fermi energies. (a)-(c) show the suppression of the Heisenberg interaction for a small Fermi energy and a strong Rashba SOC. The dependence of the periods of oscillation of the Ising interaction on the Fermi energy is depicted in (d). Other parameters are identical to those in Fig. 3.

Heisenberg interaction survives and has the range function as

$$
\mathcal{F}_{\mathrm{H}} \simeq 4\left[\gamma^{2} F_{0}\left(\gamma R_{z}\right)-\frac{C m^{2} \alpha_{R}^{2} \sin \left(2 \gamma k_{F} R_{z}\right)}{k_{F}^{4} R_{z}^{2}}\right] .
$$

The vanishing of the Ising interaction and the DM interaction can be traced back to the fact that the Rashba SOC only entangles the in-plane spin components and in-plane momentum, as schematically shown in Figs. 1(c) and 1(d). Hence, the special form of Rashba SOC leads to the intrinsic anisotropy of the RKKY interactions. One also finds that the range function with an isotropic mass has a modification quadratic in $\alpha_{R}$ compared with $F_{0}\left(R_{z}\right)$ for the conventional 3DEG. When the range function is negative, the magnetic impurities spontaneously align in parallel. Once the spin of magnetic impurities is not perpendicular to the $z$ direction, the resulting magnetization would give rise to a finite anomalous Hall current [60].

\section{CONCLUSIONS}

In summary, we have demonstrated that two kinds of linear SOCs lead to the distinct RKKY interaction, including the Heisenberg interaction, the DM interaction, and the Ising interaction. In the weak SOC limit, the Heisenberg interaction is dominant over the other two interactions in a moderately large region of parameters. The sufficiently strong SOC makes these three interactions compete with each other. We have revealed that the change in topology of the Fermi surface is accompanied by a shift in periods of oscillations of range functions. The anisotropy of RKKY interaction of the family BiTe $X$ comes from both the special Rashba SOC and the anisotropy of the effective mass. Our work provides some insights into understanding the nontrivial spin textures and paves the way for the application of $3 \mathrm{D}$ noncentrosymmetric materials in spintronics.

\section{ACKNOWLEDGMENTS}

We thank Wen-Yu Shan for useful discussions. J.Z. was supported by the Research Grant Council, University Grants Committee, Hong Kong under Grants No. 17301116 and No. C6026-16W. S.-X.W. and H.-R.C. were supported by the National Natural Science Foundation of China under Grant No. 11547200. H.-R.C. was also supported by the China Scholarship Council, the NSERC of Canada, and FQRNT of Quebec (H. Guo). We thank Compute Canada and the High Performance Computing Center of McGill University where the numerical part was done.

\section{APPENDIX A: RKKY INTERACTIONS FOR 3D NCS METALS}

In this Appendix we consider the RKKY interaction between two magnetic impurities in NCS metals. The Green's function in momentum space is given by

$$
G\left(\boldsymbol{k} ; \varepsilon+i 0^{+}\right)=\frac{1}{2} \sum_{\lambda= \pm} \frac{\sigma_{0}+\lambda \hat{\boldsymbol{k}} \cdot \boldsymbol{\sigma}}{\varepsilon-\varepsilon_{\lambda \boldsymbol{k}}+i 0^{+}},
$$

where $\hat{\boldsymbol{k}}=\boldsymbol{k} / k$, and $\varepsilon_{\lambda \boldsymbol{k}}=\frac{k^{2}}{2 m}+\lambda \alpha k$. Thus, the Green's function in real space reads

$$
\begin{aligned}
G\left( \pm \boldsymbol{R} ; \varepsilon+i 0^{+}\right)= & \frac{1}{2} \sum_{\lambda= \pm} \int \frac{\mathrm{d}^{3} \boldsymbol{k}}{(2 \pi)^{3}} \frac{\sigma_{0} \pm \lambda \hat{\boldsymbol{k}} \cdot \boldsymbol{\sigma}}{\varepsilon-\varepsilon_{\lambda \boldsymbol{k}}+i 0^{+}} \\
& \times \exp (i \boldsymbol{k} \cdot \boldsymbol{R}) .
\end{aligned}
$$

In general, the momentum $\boldsymbol{k}$ can be decomposed as $\boldsymbol{k}=$ $\boldsymbol{k}_{\|}+\boldsymbol{k}_{\perp}=(\hat{\boldsymbol{R}} \cdot \boldsymbol{k}) \hat{\boldsymbol{R}}+(\hat{\boldsymbol{R}} \times \boldsymbol{k}) \times \hat{\boldsymbol{R}}$, where $\hat{\boldsymbol{R}}=\boldsymbol{R} / R$ is the direction of $\boldsymbol{R}$ and $\boldsymbol{k} \cdot \boldsymbol{R}=k R \cos \theta$. After integrating over $\phi$, 
one finds that the part of $\boldsymbol{k}_{\perp}$ vanishes and has

$$
G\left( \pm \boldsymbol{R} ; \varepsilon+i 0^{+}\right)=G_{0} \sigma_{0} \pm G_{R} \boldsymbol{\sigma} \cdot \hat{\boldsymbol{R}},
$$

where

$$
\begin{gathered}
G_{0}=\frac{1}{2} \sum_{\lambda= \pm} \int \frac{k^{2} \sin \theta \mathrm{d} k \mathrm{~d} \theta}{(2 \pi)^{2}} \frac{\exp (i k R \cos \theta)}{\varepsilon-\varepsilon_{\lambda k}+i 0^{+}}, \\
G_{R}=\frac{1}{2} \sum_{\lambda= \pm} \int \frac{k^{2} \sin \theta \mathrm{d} k \mathrm{~d} \theta}{(2 \pi)^{2}} \frac{\lambda}{i k} \frac{\partial}{\partial R} \frac{\exp (i k R \cos \theta)}{\varepsilon-\varepsilon_{\lambda k}+i 0^{+}} .
\end{gathered}
$$

Integrating over $\theta$ yields

$$
\begin{aligned}
G_{0} & =-\frac{i}{2(2 \pi)^{2} R} \sum_{\lambda= \pm} \int_{-\infty}^{\infty} \frac{k \exp (i k R) \mathrm{d} k}{\varepsilon-\varepsilon_{\lambda k}+i 0^{+}}, \\
G_{R} & =-\frac{1}{2(2 \pi)^{2}} \frac{\partial}{\partial R}\left[\frac{1}{R} \sum_{\lambda= \pm} \lambda \int_{-\infty}^{\infty} \frac{\exp (i k R) \mathrm{d} k}{\varepsilon-\varepsilon_{\lambda k}+i 0^{+}}\right] .
\end{aligned}
$$

Note that we have used the property of even function to extend the range of integral to the whole real axis. In this way, all the remaining calculations become standard contour integrals and can be carried out immediately:

$$
\begin{aligned}
\int_{-\infty}^{\infty} \frac{k \exp (i k R) \mathrm{d} k}{\varepsilon-\varepsilon_{\lambda k}+i 0^{+}} & =-\int_{-\infty}^{\infty} \frac{2 m k \exp (i k R) \mathrm{d} k}{\left(k-k_{-}^{\lambda}+i 0^{+}\right)\left(k-k_{+}^{\lambda}-i 0^{+}\right)} \\
& =-\frac{2 m(2 \pi i) k_{+}^{\lambda} \exp \left(i k_{+}^{\lambda} R\right)}{k_{+}^{\lambda}-k_{-}^{\lambda}+i 0^{+}} \\
& =-i 2 \pi m\left(1-\frac{\lambda \zeta}{\xi+i 0^{+}}\right) \exp (i \xi-i \lambda \zeta),
\end{aligned}
$$

where $k_{ \pm}^{\lambda}= \pm \sqrt{m^{2} \alpha^{2}+2 m \varepsilon}-\lambda m \alpha, \xi=\sqrt{m^{2} \alpha^{2}+2 m \varepsilon} R$, and $\zeta=m \alpha R$. Similarly, we have

$$
\int_{-\infty}^{\infty} \frac{\exp (i k R) \mathrm{d} k}{\varepsilon-\varepsilon_{\lambda k}+i 0^{+}}=-\frac{i 2 \pi m R}{\xi+i 0^{+}} \exp (i \xi-i \lambda \zeta) .
$$

Plugging these results into Eq. (A6), we find

$$
\begin{aligned}
G_{0} & =-\frac{m \exp (i \xi)}{2 \pi R\left(\xi+i 0^{+}\right)}(\xi \cos \zeta+i \zeta \sin \zeta), \\
G_{R} & =\frac{m \exp (i \xi)}{2 \pi R\left(\xi+i 0^{+}\right)}[(i \xi-1) \sin \zeta+\zeta \cos \zeta] .
\end{aligned}
$$

Inserting the real-space Green's functions in Eq. (A3) into Eq. (2), and using the trace formulas for Pauli matrices $\operatorname{Tr}\left[\sigma_{i} \sigma_{j}\right]=2 \delta_{i j}, \operatorname{Tr}\left[\sigma_{i} \sigma_{j} \sigma_{l}\right]=2 i \varepsilon_{i j l}$, and $\operatorname{Tr}\left[\sigma_{i} \sigma_{l} \sigma_{j} \sigma_{m}\right]=$ $2\left(\delta_{i l} \delta_{j m}+\delta_{i m} \delta_{j l}-\delta_{i j} \delta_{l m}\right)$, we get

$$
H_{\mathrm{RKKY}}^{\mathrm{NCS}}=F_{\mathrm{H}} \boldsymbol{S}_{1} \cdot \boldsymbol{S}_{2}+F_{\mathrm{Ising}}\left(\boldsymbol{S}_{1} \cdot \hat{\boldsymbol{R}}\right)\left(\boldsymbol{S}_{2} \cdot \hat{\boldsymbol{R}}\right)+F_{\mathrm{DM}}\left(\boldsymbol{S}_{1} \times \boldsymbol{S}_{2}\right) \cdot \hat{\boldsymbol{R}},
$$

where the range functions are of the form

$$
\begin{aligned}
F_{\mathrm{H}} & =-\frac{2 J^{2}}{\pi} \operatorname{Im} \int_{-\infty}^{\varepsilon_{F}}\left(G_{0}^{2}+G_{R}^{2}\right) \mathrm{d} \varepsilon \\
& =\frac{J^{2} m}{2 \pi^{3} R^{4}}\left\{\cos (2 \zeta) \mathcal{I}_{2}(2)-[2 \zeta \sin (2 \zeta)+\cos (2 \zeta)-1] \mathcal{I}_{1}(2)-\frac{1}{2}\left[2 \zeta^{2} \cos (2 \zeta)+g(\zeta)\right] \mathcal{I}_{0}(2)\right\}, \\
F_{\text {Ising }}= & \frac{4 J^{2}}{\pi} \operatorname{Im} \int_{-\infty}^{\varepsilon_{F}} G_{R}^{2} \mathrm{~d} \varepsilon \\
= & \frac{J^{2} m}{2 \pi^{3} R^{4}}\left\{2[\zeta \sin (2 \zeta)+\cos (2 \zeta)-1] \mathcal{I}_{1}(2)+[1-\cos (2 \zeta)] \mathcal{I}_{2}(2)+\left[\zeta^{2}(\cos (2 \zeta)+1)+g(\zeta)\right] \mathcal{I}_{0}(2)\right\}, \\
F_{\mathrm{DM}} & =\frac{4 J^{2}}{\pi} \operatorname{Im} \int_{-\infty}^{\varepsilon_{F}} i G_{0} G_{R} \mathrm{~d} \varepsilon \\
& =\frac{-J^{2} m}{2 \pi^{3} R^{4}}\left\{\sin (2 \zeta) \mathcal{I}_{2}(2)+[2 \zeta \cos (2 \zeta)-\sin (2 \zeta)] \mathcal{I}_{1}(2)-\left[\zeta^{2} \sin (2 \zeta)+\zeta(\cos (2 \zeta)-1)\right] \mathcal{I}_{0}(2)\right\},
\end{aligned}
$$

with $\quad g(x)=-2 x \sin (2 x)-\cos (2 x)+1, \quad \xi_{F} \equiv$
$\sqrt{m^{2} \alpha^{2}+2 m \varepsilon_{F}} R . \mathcal{I}_{0,1,2}(x)$ are given in Appendix $\mathrm{C}$. Substituting $\mathcal{I}_{0,1,2}(2)$ into Eqs. (A11), (A12), and (A13), we finally obtain the range functions in Eq. (12) in the main text.

\section{APPENDIX B: APPROXIMATE RANGE FUNCTIONS FOR 3D RASHBA SEMICONDUCTORS}

In this Appendix, we calculate the RKKY interaction in 3D Rashba systems up to the corrections due to Rashba SOC up to $\alpha_{R}^{2}$. The momentum-space Green's function takes the form

$$
G\left(\boldsymbol{k} ; \varepsilon+i 0^{+}\right)=\frac{1}{2} \sum_{\lambda= \pm} \frac{\sigma_{0}+\lambda\left(\sigma \times \hat{\boldsymbol{k}}_{\|}\right)_{z}}{\varepsilon-\epsilon_{\lambda \boldsymbol{k}}+i 0^{+}},
$$

where $\boldsymbol{k}_{\|}=k_{x} \boldsymbol{e}_{x}+k_{y} \boldsymbol{e}_{y}$ denotes the in-plane component of $\boldsymbol{k}, \hat{\boldsymbol{k}}_{\|}=\boldsymbol{k}_{\|} / k_{\|}, k_{\|}=\sqrt{k_{x}^{2}+k_{y}^{2}}$ and $\epsilon_{\lambda \boldsymbol{k}}=\frac{k^{2}}{2 m}+\lambda \alpha_{R} k_{\|}$. Here we adopt the isotropic mass $m_{3}=m$ at first. 
The real-space Green's function reads

$$
\begin{aligned}
G\left( \pm \boldsymbol{R} ; \varepsilon+i 0^{+}\right)= & \frac{1}{2} \sum_{\lambda= \pm} \int \frac{\mathrm{d}^{3} \boldsymbol{k}}{(2 \pi)^{3}} \frac{\sigma_{0} \pm \lambda\left(\boldsymbol{\sigma} \times \hat{\boldsymbol{k}}_{\|}\right)_{z}}{\varepsilon-\epsilon_{\lambda \boldsymbol{k}}+i 0^{+}} \\
& \times \exp \left[i\left(\boldsymbol{k}_{\|} \cdot \boldsymbol{R}_{\|}+k_{z} \boldsymbol{R}_{z}\right)\right]
\end{aligned}
$$

where $\boldsymbol{R}=R_{x} \boldsymbol{e}_{x}+R_{y} \boldsymbol{e}_{y}+R_{z} \boldsymbol{e}_{z}$ and $\boldsymbol{R}_{\|}=R_{x} \boldsymbol{e}_{x}+R_{y} \boldsymbol{e}_{y}$ is the in-plane component of $\boldsymbol{R}$. Let us express the in-plane component of $\boldsymbol{k}$ as $\boldsymbol{k}_{\|}=\left(k_{\|} \cos \phi\right) \hat{\boldsymbol{R}}_{\|}+\left(k_{\|} \sin \phi\right) \boldsymbol{e}_{z} \times \hat{\boldsymbol{R}}_{\|}$, where $\hat{\boldsymbol{R}}_{\|}=\boldsymbol{R}_{\|} / \sqrt{R_{x}^{2}+R_{y}^{2}}$ is the in-plane direction of $\boldsymbol{R}$, $\phi$ is the relative angle between $\boldsymbol{k}_{\|}$and $\boldsymbol{R}_{\|}$. After integrating over $\phi$, one finds that the second part of $\boldsymbol{k}_{\|}$vanishes and has the real-space Green's function

$$
G\left( \pm \boldsymbol{R} ; \varepsilon+i 0^{+}\right)=G_{0} \sigma_{0} \pm G_{R}\left(\boldsymbol{\sigma} \times \hat{\boldsymbol{R}}_{\|}\right)_{z}
$$

with

$$
\begin{aligned}
G_{0}= & \frac{1}{2} \sum_{\lambda= \pm} \int \frac{k^{2} \sin \theta \mathrm{d} k \mathrm{~d} \theta}{(2 \pi)^{2}} \frac{J_{0}\left(k R_{\|} \sin \theta\right)}{\varepsilon-\epsilon_{\lambda k}+i 0^{+}} \exp \left(i k R_{z} \cos \theta\right), \\
G_{R}= & -\frac{i}{2} \frac{\partial}{\partial R_{\|}}\left[\sum_{\lambda= \pm} \lambda \int \frac{k \mathrm{~d} k \mathrm{~d} \theta}{(2 \pi)^{2}} \frac{J_{0}\left(k R_{\|} \sin \theta\right)}{\varepsilon-\epsilon_{\lambda k}+i 0^{+}}\right. \\
& \left.\times \exp \left(i k R_{z} \cos \theta\right)\right],
\end{aligned}
$$

where $J_{0}(z)$ is the zeroth-order Bessel function of the first kind, $k_{\|}=k \sin \theta$, and $k_{z}=k \cos \theta$.

Due to the complexity of the integral, we expand the Green's function as a power series of the Rashba SOC $\alpha_{R}$. The zerothorder term of $G_{0}$ takes the form

$$
G_{00}=\int \frac{k^{2} \sin \theta \mathrm{d} k \mathrm{~d} \theta}{(2 \pi)^{2}} \frac{J_{0}\left(k R_{\|} \sin \theta\right) \exp \left(i k R_{z} \cos \theta\right)}{\varepsilon-\epsilon_{k}+i 0^{+}},
$$

where $\epsilon_{k}=\frac{k^{2}}{2 m}$. The first-order term

$$
\begin{aligned}
G_{01}= & \frac{1}{2} \sum_{\lambda= \pm} \int\left(\lambda \alpha_{R} k \sin \theta\right) \frac{k^{2} \sin \theta \mathrm{d} k \mathrm{~d} \theta}{(2 \pi)^{2}} \\
& \times \frac{J_{0}\left(k R_{\|} \sin \theta\right) \exp \left(i k R_{z} \cos \theta\right)}{\left(\varepsilon-\epsilon_{k}+i 0^{+}\right)^{2}}
\end{aligned}
$$

vanishes after summing over band index $\lambda$, while the secondorder term

$$
\begin{aligned}
G_{02}= & \int\left(\alpha_{R} k \sin \theta\right)^{2} \frac{k^{2} \sin \theta \mathrm{d} k \mathrm{~d} \theta}{(2 \pi)^{2}} \\
& \times \frac{J_{0}\left(k R_{\|} \sin \theta\right) \exp \left(i k R_{z} \cos \theta\right)}{\left(\varepsilon-\epsilon_{k}+i 0^{+}\right)^{3}}
\end{aligned}
$$

is nonvanishing. One can immediately conclude that the contributions from upper and lower bands vanish all the odd-order terms of $G_{0}$. Up to the leading corrections due to Rashba SOC, we then approximate $G_{0} \simeq G_{00}+G_{02}$. On the contrary, we find that $G_{R}$ only contains odd-order terms.
Therefore, the first nonvanishing term of $G_{R}$ is linear in $\alpha_{R}$

$$
\begin{aligned}
G_{R 1}= & -i \frac{\partial}{\partial R_{\|}} \int\left(\alpha_{R} k \sin \theta\right) \frac{k \mathrm{~d} k \mathrm{~d} \theta}{(2 \pi)^{2}} \\
& \times \frac{J_{0}\left(k R_{\|} \sin \theta\right) \exp \left(i k R_{z} \cos \theta\right)}{\left(\varepsilon-\epsilon_{k}+i 0^{+}\right)^{2}} .
\end{aligned}
$$

Note that we approximate $G_{R}$ as $G_{R} \simeq G_{R 1}$. We then calculate $G_{00}, G_{02}$, and $G_{R 1}$ in a similar way. By use of Eq. (C8), integrating over $\theta$ leads to

$$
G_{00}=-\frac{i}{(2 \pi)^{2} R} \int_{-\infty}^{\infty} \frac{k \exp (i k R) \mathrm{d} k}{\varepsilon-\epsilon_{k}+i 0^{+}},
$$

where $R=\sqrt{R_{\|}^{2}+R_{z}^{2}}$ and the last step is similar to Eq. (A6). Carrying out the straightforward contour integral, we have

$$
G_{00}=-\frac{m \exp \left(i k_{\varepsilon} R\right)}{2 \pi R},
$$

where $k_{\varepsilon}=\sqrt{2 m \varepsilon}$. For $G_{R 1}$, the integration over $\theta$ is the same as that of $G_{00}$ in Eq. (B5). Hence one immediately gets

$$
G_{R 1}=-\frac{\alpha_{R}}{(2 \pi)^{2}} \frac{\partial}{\partial R_{\|}}\left[\frac{1}{R} \int_{-\infty}^{\infty} \frac{k \exp (i k R) \mathrm{d} k}{\left(\varepsilon-\epsilon_{k}+i 0^{+}\right)^{2}}\right] .
$$

Performing the standard contour integral, we arrive at the result

$$
G_{R 1}=\frac{i m R_{\|} \exp \left(i k_{\varepsilon} R\right)}{2 \pi R^{2}} \zeta^{\prime},
$$

where the dimensionless parameter $\zeta^{\prime}=m \alpha_{R} R$. The last one, $G_{02}$, can be calculated similarly. Using Eq. (C11), we integrate over $\theta$ and get

$$
\begin{aligned}
G_{02}= & -\frac{i \alpha_{R}^{2}}{(2 \pi)^{2}}\left\{\frac{1}{R} \int_{-\infty}^{\infty} \frac{k^{3} \exp (i k R) \mathrm{d} k}{\left(\varepsilon-\epsilon_{k}+i 0^{+}\right)^{3}}\right. \\
& \left.+\frac{\partial^{2}}{\partial R_{z}^{2}}\left[\frac{1}{R} \int_{-\infty}^{\infty} \frac{k \exp (i k R) \mathrm{d} k}{\left(\varepsilon-\epsilon_{k}+i 0^{+}\right)^{3}}\right]\right\} .
\end{aligned}
$$

After some algebra, we have

$$
G_{02}=-\frac{m \exp \left(i k_{\varepsilon} R\right)}{2 \pi R}\left(\frac{i}{k_{\varepsilon} R+i 0^{+}}-\frac{R_{\|}^{2}}{2 R^{2}}\right) \zeta^{\prime 2} .
$$

Collecting the results in Eqs. (B10), (B12), and (B14), we have the following approximations:

$$
\begin{aligned}
G_{0} & \simeq-\frac{m \exp \left(i k_{\varepsilon} R\right)}{2 \pi R}\left[1+\left(\frac{i}{k_{\varepsilon} R+i 0^{+}}-\frac{R_{\|}^{2}}{2 R^{2}}\right) \zeta^{\prime 2}\right], \\
G_{R} & \simeq \frac{i m R_{\|} \exp \left(i k_{\varepsilon} R\right)}{2 \pi R^{2}} \zeta^{\prime} .
\end{aligned}
$$

Since the real-space Green's function in Eq. (B3) has the same structure as that in Eq. (A3), the RKKY interaction in 3D Rashba systems takes a similar form:

$$
\begin{aligned}
H_{\mathrm{RKKY}}^{3 \mathrm{DR}}= & \mathcal{F}_{\mathrm{H}} \boldsymbol{S}_{1} \cdot \boldsymbol{S}_{2} \\
& +\mathcal{F}_{\mathrm{DM}}\left[\left(\boldsymbol{S}_{1} \times \boldsymbol{S}_{2}\right)_{x} \sin \varphi-\left(\boldsymbol{S}_{1} \times \boldsymbol{S}_{2}\right)_{y} \cos \varphi\right] \\
& +\mathcal{F}_{\mathrm{Ising}}\left(S_{1}^{x} \sin \varphi-S_{1}^{y} \cos \varphi\right)\left(S_{2}^{x} \sin \varphi-S_{2}^{y} \cos \varphi\right),
\end{aligned}
$$


where $\varphi=\arctan \left(R_{y} / R_{x}\right)$ and the ranges functions are given as

$$
\begin{aligned}
\mathcal{F}_{\mathrm{H}} & =-\frac{2 J^{2}}{\pi} \operatorname{Im} \int_{-\infty}^{\varepsilon_{F}}\left(G_{0}^{2}+G_{R}^{2}\right) \mathrm{d} \varepsilon, \\
\mathcal{F}_{\text {Ising }} & =\frac{4 J^{2}}{\pi} \operatorname{Im} \int_{-\infty}^{\varepsilon_{F}} G_{R}^{2} \mathrm{~d} \varepsilon, \\
\mathcal{F}_{\mathrm{DM}} & =\frac{4 J^{2}}{\pi} \operatorname{Im} \int_{-\infty}^{\varepsilon_{F}} i G_{0} G_{R} \mathrm{~d} \varepsilon .
\end{aligned}
$$

Plugging Eq. (B15) into Eq. (B17) and using the basic integrals in Eqs. (C9) and (C10), we obtain the following approximations up to the leading corrections from SOC:

$$
\begin{aligned}
\mathcal{F}_{\mathrm{H}} & \simeq 4 F_{0}(R)-8 m^{2} \alpha_{R}^{2}\left[R_{\|}^{2} F_{0}(R)+\frac{C \sin \left(2 k_{F} R\right)}{2 k_{F}^{4} R^{2}}\right], \\
\mathcal{F}_{\text {Ising }} & \simeq 8 m^{2} \alpha_{R}^{2} R_{\|}^{2} F_{0}(R), \\
\mathcal{F}_{\mathrm{DM}} & \simeq-8 m \alpha_{R} R_{\|} F_{0}(R),
\end{aligned}
$$

where $F_{0}(R)$ is the range function for $3 \mathrm{DEG}$ in Eq. (14).

The impacts of anisotropy of the effective mass $\gamma=$ $\sqrt{m_{3} / m} \neq 1$ in Eq. (16) can be encoded into the RKKY interaction through a transformation introduced in Ref. [34]. Specifically, the real-space Green's functions for the isotropic Hamiltonian and the anisotropic one can be connected by the following transformation $\left(k_{z}, R_{z}\right) \rightarrow\left(\gamma k_{z}, R_{z} / \gamma\right)$. We note that the above transformation preserves the volume of the Fermi sphere. After some algebra, one gets the relation $\tilde{G}\left(R_{x}, R_{y}, R_{z}\right)=\gamma G\left(R_{x}, R_{y}, \gamma R_{z}\right)$, leading to the anisotropic RKKY interaction

$$
\tilde{H}_{\mathrm{RKKY}}\left(R_{x}, R_{y}, R_{z}\right)=\gamma^{2} H_{\mathrm{RKKY}}\left(R_{x}, R_{y}, \gamma R_{z}\right),
$$

which implies that the anisotropic effective mass may result in an anisotropic RKKY interaction. Thus, it is easy to reach the approximate range functions in Eq. (19).

\section{APPENDIX C: SOME INTEGRAL FORMULAS}

In this Appendix we evaluate two sets of frequently used integrals $\mathcal{I}_{n}(x)$ and $\mathcal{J}_{n}$ :

$$
\begin{gathered}
\mathcal{I}_{n}(x)=\operatorname{Im} \int_{-\infty}^{\xi_{F}} \frac{i^{n} \exp (i x \xi)}{\left(\xi+i 0^{+}\right)^{1-n}} \mathrm{~d} \xi \\
\mathcal{J}_{n}=\int_{0}^{\pi}(\sin \theta)^{2 n+1} J_{0}\left(k R_{\|} \sin \theta\right) \exp \left(i k R_{z} \cos \theta\right) \mathrm{d} \theta,
\end{gathered}
$$

with $n$ being a nonnegative integer. For $n>1, \mathcal{I}_{n}(x)$ and $\mathcal{J}_{n}$ can be generated by $\mathcal{I}_{0}(x)$ and $\mathcal{J}_{0}$, respectively.

$$
\begin{gathered}
\mathcal{I}_{n}(x)=\frac{\partial^{n}}{\partial x^{n}}\left[\operatorname{Im} \int_{-\infty}^{\xi_{F}} \frac{\exp (i x \xi)}{\xi+i 0^{+}} \mathrm{d} \xi\right]=\frac{\partial^{n} \mathcal{I}_{0}(x)}{\partial x^{n}}, \\
\mathcal{J}_{n}=\sum_{m=0}^{n} \frac{C_{n}^{m}}{k^{2 m}} \frac{\partial^{2 m} \mathcal{J}_{0}}{\partial R_{z}^{2 m}},
\end{gathered}
$$

where $C_{n}^{m}=\frac{n !}{m !(n-m) !}$ is the binomial coefficient. Note that the infinitesimal $0^{+}$in the numerator in Eq. (C3) can be safely omitted. Therefore, our task is to compute $\mathcal{I}_{0}(x)$ and $\mathcal{J}_{0}$.

Let us first deal with $\mathcal{I}_{0}(x)$ as follows:

$$
\begin{aligned}
\mathcal{I}_{0}(x) & =\operatorname{Im} \int_{-\infty}^{\xi_{F}} \frac{\exp (i x \xi)}{\xi+i 0^{+}} \mathrm{d} \xi \\
& =\operatorname{Im}\left[\mathcal{P} \int_{-\infty}^{\xi_{F}} \frac{\exp (i x \xi)}{\xi} \mathrm{d} \xi-i \pi \int_{-\infty}^{\xi_{F}} \exp (i x \xi) \delta(\xi) \mathrm{d} \xi\right] \\
& =\frac{\pi}{2} \operatorname{sgn}(x)+\operatorname{Si}\left(x \xi_{F}\right)-\pi,
\end{aligned}
$$

where we have used the identity $\frac{1}{x+i 0^{+}}=\mathcal{P}\left(\frac{1}{x}\right)-i \pi \delta(x)$, with $\mathcal{P}$ denoting the Cauchy principle value. $\operatorname{Si}(z)=\int_{0}^{z} \mathrm{~d} t \sin t / t$ is the sine integral [61]. Since only the case $x>0$ is of interest, we have

$$
\mathcal{I}_{0}(x)=\frac{\pi}{2}+\operatorname{Si}\left(x \xi_{F}\right)-\pi=\operatorname{si}\left(x \xi_{F}\right)
$$

with $\operatorname{si}(z)=-\int_{z}^{\infty} \mathrm{d} t \sin t / t=\operatorname{Si}(z)-\pi / 2$. Next, we turn to evaluate $\mathcal{J}_{0}$ :

$$
\begin{aligned}
\mathcal{J}_{0} & =\int_{0}^{\pi} \sin \theta J_{0}\left(k R_{\|} \sin \theta\right) \exp \left(i k R_{z} \cos \theta\right) \mathrm{d} \theta \\
& =-\int_{0}^{\pi} J_{0}\left(k R_{\|} \sqrt{1-\cos ^{2} \theta}\right) \exp \left(i k R_{z} \cos \theta\right) \mathrm{d}(\cos \theta) \\
& =\int_{-1}^{1} J_{0}\left(k R_{\|} \sqrt{1-t^{2}}\right) \exp \left(i k R_{z} t\right) \mathrm{d} t \\
& =2 \int_{0}^{1} J_{0}\left(k R_{\|} \sqrt{1-t^{2}}\right) \cos \left(k R_{z} t\right) \mathrm{d} t
\end{aligned}
$$

Using the formula in Ref. [61], we get

$$
\mathcal{J}_{0}=\frac{2 \sin (k R)}{k R},
$$

where $R=\sqrt{R_{\|}^{2}+R_{z}^{2}}$. From Eqs. (C3) and (C4), we have

$$
\begin{gathered}
\mathcal{I}_{1}(x)=\frac{\partial \operatorname{si}\left(x \xi_{F}\right)}{\partial x}=\frac{\sin \left(x \xi_{F}\right)}{x} \\
\mathcal{I}_{2}(x)=\frac{\partial^{2} \operatorname{si}\left(x \xi_{F}\right)}{\partial x^{2}}=\frac{\xi_{F} \cos \left(x \xi_{F}\right)}{x}-\frac{\sin \left(x \xi_{F}\right)}{x^{2}}
\end{gathered}
$$

and

$$
\mathcal{J}_{1}=\left(1+\frac{1}{k^{2}} \frac{\partial^{2}}{\partial R_{z}^{2}}\right) \frac{2 \sin (k R)}{k R}
$$

which are used in Appendices A and B. Those who are interested in higher-order corrections from SOC need to continue to look for the expressions of larger $n$. 
[1] Y. A. Bychkov and E. I. Rashba, J. Phys. C 17, 6039 (1984).

[2] R. Winkler, Spin-orbit Coupling Effects in Two-Dimensional Electron and Hole Systems (Springer Science \& Business Media, 2003), Vol. 191.

[3] S. LaShell, B. A. McDougall, and E. Jensen, Phys. Rev. Lett. 77, 3419 (1996).

[4] A. Ohtomo and H. Y. Hwang, Nature 427, 423 (2004).

[5] A. D. Caviglia, M. Gabay, S. Gariglio, N. Reyren, C. Cancellieri, and J.-M. Triscone, Phys. Rev. Lett. 104, 126803 (2010).

[6] M. Ben Shalom, M. Sachs, D. Rakhmilevitch, A. Palevski, and Y. Dagan, Phys. Rev. Lett. 104, 126802 (2010).

[7] P. D. C. King, S. M. Walker, A. Tamai, A. De La Torre, T. Eknapakul, P. Buaphet, S.-K. Mo, W. Meevasana, M. S. Bahramy, and F. Baumberger, Nat. Commun. 5, 3414 (2014).

[8] G. Khalsa, B. Lee, and A. H. MacDonald, Phys. Rev. B 88, 041302 (2013).

[9] J. Zhou, W.-Y. Shan, and D. Xiao, Phys. Rev. B 91, 241302 (2015).

[10] A. Fert, V. Cros, and J. Sampaio, Nat. Nano. 8, 152 (2013).

[11] A. Manchon, H. C. Koo, J. Nitta, S. M. Frolov, and R. A. Duine, Nat. Mater. 14, 871 (2015).

[12] J. Sinova, S. O. Valenzuela, J. Wunderlich, C. H. Back, and T. Jungwirth, Rev. Mod. Phys. 87, 1213 (2015).

[13] A. Soumyanarayanan, N. Reyren, A. Fert, and C. Panagopoulos, Nature 539, 509 (2016).

[14] I. Dzyaloshinsky, J. Phys. Chem. Solids 4, 241 (1958).

[15] T. Moriya, Phys. Rev. 120, 91 (1960).

[16] M. A. Ruderman and C. Kittel, Phys. Rev. 96, 99 (1954).

[17] T. Kasuya, Prog. Theor. Phys. 16, 45 (1956).

[18] K. Yosida, Phys. Rev. 106, 893 (1957).

[19] M. Liebmann, C. Rinaldi, D. Di Sante, J. Kellner, C. Pauly, R. N. Wang, J. E. Boschker, A. Giussani, S. Bertoli, M. Cantoni, L. Baldrati, M. Asa, I. Vobornik, G. Panaccione, D. Marchenko, J. Sánchez-Barriga, O. Rader, R. Calarco, S. Picozzi, R. Bertacco, and M. Morgenstern, Adv. Mater. 28, 560 (2016).

[20] H. J. Elmers, R. Wallauer, M. Liebmann, J. Kellner, M. Morgenstern, R. N. Wang, J. E. Boschker, R. Calarco, J. Sánchez-Barriga, O. Rader, D. Kutnyakhov, S. V. Chernov, K. Medjanik, C. Tusche, M. Ellguth, H. Volfova, S. Borek, J. Braun, J. Minár, H. Ebert, and G. Schönhense, Phys. Rev. B 94, 201403 (2016).

[21] K. Ishizaka, M. Bahramy, H. Murakawa, M. Sakano, T. Shimojima, T. Sonobe, K. Koizumi, S. Shin, H. Miyahara, A. Kimura et al., Nat. Mater. 10, 521 (2011).

[22] M. Sakano, M. S. Bahramy, A. Katayama, T. Shimojima, H. Murakawa, Y. Kaneko, W. Malaeb, S. Shin, K. Ono, H. Kumigashira, R. Arita, N. Nagaosa, H. Y. Hwang, Y. Tokura, and K. Ishizaka, Phys. Rev. Lett. 110, 107204 (2013).

[23] J. Kang and J. Zang, Phys. Rev. B 91, 134401 (2015).

[24] V. P. Mineev and Y. Yoshioka, Phys. Rev. B 81, 094525 (2010).

[25] G. Landolt, S. V. Eremeev, Y. M. Koroteev, B. Slomski, S. Muff, T. Neupert, M. Kobayashi, V. N. Strocov, T. Schmitt, Z. S. Aliev, M. B. Babanly, I. R. Amiraslanov, E. V. Chulkov, J. Osterwalder, and J. H. Dil, Phys. Rev. Lett. 109, 116403 (2012).

[26] H. Maaß, H. Bentmann, C. Seibel, C. Tusche, S. V. Eremeev, T. R. F. Peixoto, O. E. Tereshchenko, K. A. Kokh, E. V. Chulkov, J. Kirschner, and F. Reinert, Nat. Commun. 7, 11621 (2016).
[27] M. Uchida, Y. Onose, Y. Matsui, and Y. Tokura, Science 311, 359 (2006).

[28] U. K. Roszler, A. N. Bogdanov, and C. Pfleiderer, Nature 442, 797 (2006).

[29] S. Mühlbauer, B. Binz, F. Jonietz, C. Pfleiderer, A. Rosch, A. Neubauer, R. Georgii, and P. Böni, Science 323, 915 (2009).

[30] X. Z. Yu, Y. Onose, N. Kanazawa, J. H. Park, J. H. Han, Y. Matsui, N. Nagaosa, and Y. Tokura, Nature 465, 901 (2010).

[31] S. S. P. Parkin, N. More, and K. P. Roche, Phys. Rev. Lett. 64, 2304 (1990).

[32] T. Jungwirth, J. Sinova, J. Mašek, J. Kučera, and A. H MacDonald, Rev. Mod. Phys. 78, 809 (2006).

[33] The $s-d$ interaction is widely used for the RKKY interaction among magnetic impurities in metals, semimetals, and doped semiconductors. Essentially, the RKKY interaction is a secondorder perturbation of the $s$ - $d$ interaction and thus is valid only in the weak-coupling limit $J S / W \ll 1$, where $W$ is the bandwidth of conduction electrons, $S$ refers to the quantum number of the spins of magnetic impurities in Eq. (1) in the main text. The systematical treatments in the intermediate- and strong-coupling limits are reviewed in Ref. [32].

[34] H.-R. Chang, J. Zhou, S.-X. Wang, W.-Y. Shan, and D. Xiao, Phys. Rev. B 92, 241103 (2015).

[35] D. N. Aristov, Phys. Rev. B 55, 8064 (1997).

[36] V. I. Litvinov and V. K. Dugaev, Phys. Rev. B 58, 3584 (1998).

[37] M. V. Hosseini and M. Askari, Phys. Rev. B 92, 224435 (2015).

[38] D. Mastrogiuseppe, N. Sandler, and S. E. Ulloa, Phys. Rev. B 93, 094433 (2016).

[39] H. Imamura, P. Bruno, and Y. Utsumi, Phys. Rev. B 69, 121303 (2004).

[40] A. Schulz, A. De Martino, P. Ingenhoven, and R. Egger, Phys. Rev. B 79, 205432 (2009).

[41] J.-J. Zhu, K. Chang, R.-B. Liu, and H.-Q. Lin, Phys. Rev. B 81, 113302 (2010).

[42] J. Klinovaja and D. Loss, Phys. Rev. B 87, 045422 (2013).

[43] P. Lyu, N.-N. Liu, and C. Zhang, J. Appl. Phys. 102, 103910 (2007).

[44] P. Simon, B. Braunecker, and D. Loss, Phys. Rev. B 77, 045108 (2008).

[45] H.-H. Lai, W.-M. Huang, and H.-H. Lin, Phys. Rev. B 79, 045315 (2009).

[46] S. Chesi and D. Loss, Phys. Rev. B 82, 165303 (2010).

[47] T. Kernreiter, M. Governale, and U. Zülicke, Phys. Rev. Lett. 110, 026803 (2013).

[48] We have neglected the $k$-cubic SOC for $B_{20}$ compounds, which turns out to be crucial to anisotropic magnetotransport but not to the chiral spin textures [23].

[49] K. V. Samokhin, Phys. Rev. B 78, 144511 (2008).

[50] D. Xiao, M.-C. Chang, and Q. Niu, Rev. Mod. Phys. 82, 1959 (2010).

[51] X. Wan, A. M. Turner, A. Vishwanath, and S. Y. Savrasov, Phys. Rev. B 83, 205101 (2011).

[52] I. L. Aleiner and V. I. Fal'ko, Phys. Rev. Lett. 87, 256801 (2001).

[53] M. S. Bahramy, R. Arita, and N. Nagaosa, Phys. Rev. B 84, 041202 (2011).

[54] S. Maiti, V. Zyuzin, and D. L. Maslov, Phys. Rev. B 91, 035106 (2015). 
[55] F.-X. Xiang, X.-L. Wang, M. Veldhorst, S.-X. Dou, and M. S. Fuhrer, Phys. Rev. B 92, 035123 (2015).

[56] L. Ye, J. G. Checkelsky, F. Kagawa, and Y. Tokura, Phys. Rev. B 91, 201104 (2015).

[57] T. Ideue, L. Ye, J. G. Checkelsky, H. Murakawa, Y. Kaneko, and Y. Tokura, Phys. Rev. B 92, 115144 (2015).

[58] In numerical calculations, we adopt the finite-temperature formalism for the RKKY interaction in Ref. [45] and take $m=0.1 m_{e}, m_{3}=0.5 m_{e}, T=5.0 \mathrm{~K}, R_{x}=R_{z} \neq 0$, and $R_{y}=0$, where $m_{e}$ is the electron rest mass. In addition, all the range functions are scaled by a common parameter $C$ at $\varepsilon_{F}=0.1 \mathrm{eV}$.

[59] H. Murakawa, M. Bahramy, M. Tokunaga, Y. Kohama, C. Bell, Y. Kaneko, N. Nagaosa, H. Hwang, and Y. Tokura, Science 342, 1490 (2013).

[60] D. Culcer, A. MacDonald, and Q. Niu, Phys. Rev. B 68, 045327 (2003).

[61] I. Gradshteyn and I. Ryzhik, Table of Integrals, Series, and Products (Elsevier Science, 2007). 